Écrire

l'histoire

\section{Écrire l'histoire}

Histoire, Littérature, Esthétique

12 | 2013

Présent (2)

\title{
Le présent au contemporain
}

Dominique VIART, Gianfranco RUBINo (dir.), Écrire le présent (2013)

\section{Carole Furmanek}

\section{(2) OpenEdition}

1 Journals

Édition électronique

URL : http://journals.openedition.org/elh/353

DOI : $10.4000 /$ elh.353

ISSN : 2492-7457

Éditeur

CNRS Éditions

\section{Édition imprimée}

Date de publication : 15 novembre 2013

Pagination : 112-114

ISBN : 978-2-35698-065-6

ISSN : 1967-7499

Référence électronique

Carole Furmanek, «Le présent au contemporain », Écrire l'histoire [En ligne], 12 | 2013, mis en ligne le 15 novembre 2016, consulté le 23 septembre 2020. URL : http://journals.openedition.org/elh/353 ;

DOI : https://doi.org/10.4000/elh.353

Ce document a été généré automatiquement le 23 septembre 2020.

Tous droits réservés 


\title{
Le présent au contemporain
}

\author{
Dominique VIART, Gianfranco RUBINO (dir.), Écrire le présent (2013)
}

\section{Carole Furmanek}

\section{RÉFÉRENCE}

Dominique VIART, Gianfranco RUBINo (dir.), Écrire le présent, Paris, A. Colin (Recherches), 2013, $248 \mathrm{p}$.

1 Quelles formes littéraires aujourd'hui pour le présent, ce temps si éphémère? Comment la littérature répond-elle à l'actualité brûlante, déjà mise en récit, en images? Cette discussion est d'autant plus pertinente qu'une opinion répandue semble vouer la littérature contemporaine au passé, à ses troubles comme à sa mélancolie. Toutes ces questions trouvent une réponse intéressante et riche dans l'ouvrage Écrire le présent, qui a l'audace de rendre la littérature contemporaine au présent. Étudier ce que la littérature d'aujourd'hui écrit à propos d'aujourd'hui, analyser de quelles manières spécifiques elle relaie, transforme, interprète les appels de notre présent : voilà le défi que s'est fixé cette grande étude collective dirigée par Dominique Viart et Gianfranco Rubino, fruit d'un séminaire réunissant deux des plus anciennes équipes engagées dans la recherche sur la littérature contemporaine ${ }^{1}$. Cette équipe internationale ${ }^{2}$ a réorienté son travail, jusqu'alors tourné vers l'étude du poids du passé, collectif et personnel, vers une autre approche de la réalité, en prenant à bras-le-corps cette question décisive $\mathrm{du}$ " présent $^{3}$ ». Les deux colloques sur les « Fins de la littérature ${ }^{4}$ » avaient déjà suscité un intérêt fort pour les régimes d'historicité. Comprendre en quoi la littérature française de notre temps serait présentiste est une question qui s'est imposée très logiquement pour ce groupe de chercheurs. Cette piste a ouvert la voie à des investigations plus larges sur les figurations du présent.

2 Écrire le présent est un livre qui aborde avec précision les œuvres d'un bon nombre d'écrivains contemporains : Pierre Michon, Annie Ernaux (sous deux angles différents), Bertrand Leclaire, Laurent Mauvignier, Jean-Louis Gutierres, Salim Bachi, Frédéric Beigbeder, Slimane Benaïssa, Maurice G. Dantec, Didier Goupil, Luc Lang, Marc-Édouard 
Nabe, Marc Weitzmann, Patrick Bouvet, Martine Sonnet, Gérard Mordillat, Michel Houellebecq, François Bon, Leslie Kaplan, Thierry Beinstingel, Élisabeth Filhol, Maylis de Kerangal, Thierry Jonquet, Régis Jauffret, Sylvie Germain, Philippe Claudel, Antoni Casas Ros, Éric Chevillard et Jean-Philippe Toussaint. Si ces approches individuelles donnent tout leur prix et tout leur sens à un travail qui veut modéliser avec précision les pratiques actuelles, ces études trouvent leur place dans cinq perspectives de recherche plus larges, essentielles pour repérer synthétiquement les grands enjeux de la littérature au présent.

3 Ainsi s'intéresse-t-on d'abord, et sans contradiction, aux " Rémanences de l'Histoire ", autant dire à l'étrange actualité de questions historiques non résolues, des questions que les auteurs destinent expressément à leurs contemporains et qui permettent clairement d'interroger les limites temporelles de notre «aujourd'hui » et de révéler l'absence ressentie au sein de ce présent.

4 Puis vient le moment de la confrontation à l'événement, cet événement terrorisant, véritablement extraordinaire, comme ont pu l'être les attentats du 11 septembre 2001, pris tout à la fois comme matériau et comme défi lancé à la littérature. Si la représentation artistique de cet événement apparaît d'emblée être une gageure, les auteurs contemporains, confrontés au déferlement de médiatisations audiovisuelles de l'événement en direct, en viennent aussi à interroger le rôle et la place à accorder à la fiction dans leurs œuvres et sont soucieux de repenser leur art au vu de cette concomitance entre l'événement et ses décisives et obsédantes représentations médiatiques.

5 Dépassant la perspective chronologique des dates marquantes pour une perspective d'ensemble qui cerne notre époque, la troisième partie interroge le " Présent social ». Les romanciers contemporains n'hésitent pas à s'engager sur la voie de mises en forme littéraires du travail tel qu'il s'exerce aujourd'hui, ce qui aboutit à une véritable «sociologisation » du roman. Ce brouillage des frontières disciplinaires n'est certes pas inédit en littérature (qu'on pense à Zola, à Balzac), mais son retour dans la littérature contemporaine intéresse aussi bien les historiens, les sociologues que les littéraires, car il oblige à repenser les interactions entre ces disciplines scientifiques et artistiques, mais aussi parce qu'il repose la question du réalisme.

6 La quatrième partie, consacrée à l'analyse des «Sentiments du présent ", illustre la diversité des réponses à cette même question, cette même tension du quotidien. Si le présent s'efface, se dissout chez Philippe Claudel et Sylvie Germain, il apparaît impossible chez Éric Chevillard, mais ressuscite chez Antoni Casas Ros, qui l'offre à la palingénésie. Le quotidien reste irrémédiablement tenu à distance pour Régis Jauffret.

7 Pour la dernière partie, "Écrire aujourd'hui », Jean-Philippe Toussaint répond librement aux questions de Dominique Viart sur le double geste artistique qui consiste à écrire le présent et à créer du présent.

8 On ne peut que saluer cette ambition de cerner un objet théorique aussi fugitif. Qu'estce donc que le présent ? Catégorie temporelle à la délimitation impossible, le présent ne semble pas déterminer de sujet particulier, même s'il est patent que les romanciers contemporains développent leur intérêt pour quelques aspects caractéristiques de l'époque: le monde du travail, les problèmes sociaux et les intrigues politiques trouvent leur place dans l'écriture du présent. Représenter des situations extrêmes vécues lors de grands événements internationaux, que ce soient les attentats du 11Septembre ou les ravages de l'ouragan Katrina, importe. Cette pression de l'actualité 
intéresse les auteurs contemporains. Pourtant l'ambition de saisir le quotidien dans sa banalité et sa complexité n'a jamais disparu : comme l'analyse Écrire le présent, le roman contemporain continue à s'alimenter de faits divers et d'inventaires de lieux et de choses. Le présent serait autant l'immédiat que le quotidien ou l'événement. L'écriture littéraire du présent se confondrait parfois avec l'analyse sociologique de ce qui nous entoure. Il est donc tout à fait profitable que les études soient accompagnées d'un avant-propos et d'une introduction qui parviennent à dessiner clairement des lignes de force sans atténuer la complexité de l'objet et la pluralité des démarches artistiques. Dominique Viart s'inspire de l'analyse de Roland Barthes - si attaché à la notation et au haïku - lorsqu'il envisage une écriture du présent. Il propose une condition ferme et convaincante à sa définition: le présent n'existe «que pris dans une Histoire, qui lui donne place et sens ${ }^{5}$ ». La distinction à opérer entre le présent et la notion de modernité attachée au fugitif et au transitoire fait l'objet d'une analyse d'un grand intérêt. Le manque d'approche philosophique de la notion - remarqué par Dominique Viart - est caractéristique de la manière dont la littérature s'est rapprochée des sciences humaines. La question, essentielle, de savoir si un rapport direct entre le présent et sa représentation littéraire n'est pas devenu impossible - considérant le flux permanent d'images qui nous entourent-constitue un des enjeux majeurs de l'ouvrage. Maintenant que le rapport de l'homme à l'événement s'accompagne de sa médiatisation, la tâche de la littérature change-t-elle?

Le livre aborde aussi en filigrane la question de l'essence intempestive ou inactuelle de la littérature qui viendrait justement contrer l'époque et transformer les relations entre passé, présent et futur. Discrétion calculée qui évite, sans doute, de réitérer ce que Giorgio Agamben avait déjà noté avec finesse dans son livre Qu'est-ce que le contemporain ${ }^{6}$. Mais discrétion qui ne manque pas d'accréditer la thèse de ce nouveau régime d'historicité "présentiste » qui nous concernerait. L'interrogation actuelle du présent est bien liée aux retours du sujet et du réel, qu'une histoire de la littérature contemporaine raconte déjà ${ }^{7}$.

\section{NOTES}

1. Le Laboratorio di ricerche sul contemporaneo (LARC) de l'université de Rome La Sapienza et l'équipe Analyses littéraires et histoire de la langue (ALITHILA) de l'université Lille-III.

2. Elle est composée d'Annalisa Bertoni, Alexandre Bleau, Carine Capone, Jean-Max Colard, Flavia Conti, Valerio Cordiner, Chetro De Carolis, Laurent Demanze, Marie-Pascale Huglo, Mario Maccherini, Mélanie Lamarre, Annie Oliver, Gianfranco Rubino, Flavio Sorrentino et Dominique Viart.

3. Gianfranco Rubino (dir.), Voix du contemporain. Histoire, mémoire et réel dans le roman français d'aujourd'hui, Rome, Bulzoni, 2006 ; id., Présences du passé dans le roman français contemporain, Rome, Bulzoni, 2007 ; Dominique Viart (dir.), Écritures contemporaines 10. "Nouvelles Écritures littéraires de l'histoire ", Caen, Lettres modernes Minard, 2009.

4. Dominique Viart, Laurent Demanze (dir.), Fins de la littérature, Armand Colin (Recherches), 2012, t. I, Esthétiques et discours de la fin, t. II, Historicité de la littérature contemporaine. 
5. Dominique Viart, Gianfranco Rubino (dir.), op. cit., p. 17.

6. Giorgio Agamben, Qu'est-ce que le contemporain?, trad. de l'italien par Maxime Rovere, Payot \& Rivages, 2008. Voir aussi Martin Rueff, «La concordance des temps », dans Qu'est-ce que le contemporain?, textes réunis par Lionel Ruffel, Nantes, Cécile Defaut, 2010, p. 93-110.

7. Voir Dominique Viart, Bruno Vercier, La Littérature française au présent. Héritage, modernité, mutations [2005], Bordas, 2008, $2^{\mathrm{e}}$ éd. augm.

\section{INDEX}

oeuvrecitee Écrire le présent - (Dominique Viart, Gianfranco Rubino (dir.), 2013)

\section{AUTEURS}

\section{CAROLE FURMANEK}

Agrégée de lettres modernes et docteur ès lettres, Carole Furmanek a soutenu la thèse intitulée À la recherche d'une communauté poétique ? Constitutions de rituels et dissolutions de groupes, du Tombeau de Théophile Gautier à la mort de Mallarmé (1872-1898). Afin de réinterroger la poésie d'après 1870 dans ses relations avec le politique et le religieux, elle y formule l'hypothèse d'une communauté poétique et remet en cause la doxa du poète solitaire. Membre de l'équipe Littérature et civilisation du XIX ${ }^{e}$ siècle du CÉRILAC (Paris 7) et rédactrice d'un carnet de recherche sur l'archive au XIX ${ }^{\mathrm{e}}$ siècle, elle a publié des articles sur la poésie postromantique. Ancienne ATER de l'université Lille-III, elle enseigne actuellement au lycée Gambetta-Carnot à Arras. 